\title{
Mathematical models for estimating the degree of influence of major factors on performance and accuracy of coordinate measuring machines
}

\author{
Alexander F. Rezchikov ${ }^{1}$, Andrei V. Kochetkov², and Oleg V. Zakharov,3,"* \\ ${ }^{1}$ Institute of Precision Mechanics and Control of RAS, 24 Working st., Saratov, 410028, Russia \\ ${ }^{2}$ Perm National Research Polytechnic University, 29 Komsomol pr., Perm, 614990, Russia \\ ${ }^{3}$ Yuri Gagarin State Technical University, 77 Politecknicheskaya str., Saratov, 410054, Russia \\ ${ }^{4}$ Samara National Research University, 34 Moscow Highway, Samara, 443086, Russia
}

\begin{abstract}
Increased productivity of Coordinate Measuring Machines, while providing given level of accuracy, is complex and actual problem of robotics and metrology. Therefore, this article provides a set of mathematical models that allow for prediction of values for performance and accuracy of advanced Coordinate Measuring Machines based on system dynamics method. The analysis of scientific literature and statistical data of exploitation CMMs Global Performance (DEA, Italy), CMM-750 (Lapik, Russia), FARO Arm 9 (FARO Technologies, USA) has identified 25 major factors of the process control and their mutual impact. Of the 16 basic factors are internal system parameters, 6 input actions and 3 external factors. As a result of the system analysis of the research object identifies the main characteristics of coordinate measuring machines that affect performance and measurement accuracy. We have done a general graph of causal relationships between modeled characteristics, on the basis of which is made up and numerically solved a system of nonlinear differential equations. In developing this system of equations is used in statistical data describing the cause-effect relationship between the internal model parameters and environmental factors. These solutions make it possible to practically interpret developed models and methods of system dynamics used in solving the problem.
\end{abstract}

\section{Introduction}

In developed countries, high-tech production, primarily the defense complex, use coordinate measuring machines (CMM). By the beginning of the XXI century in the world, operating more than 500,000 CMM. One third of this quantity is used in the United States, about the same in Germany, Great Britain and Japan. Active industrial development in China, South Korea, India, Brazil, is also accompanied by the rapid implementation of CMM. Complete CMM produced by only a few companies in the world.

The basis of most of the known of working capacity CMMs laid implementation of the Cartesian coordinate system. This feature has become a bottleneck in the way of a new generation of CMMs. Options traditional threeaxis CMM when measuring complex spatial surfaces is not enough. An important breakthrough in this direction was the creation of the six-axis CMM Saratov group of experts, which included engineers, physicist's optics, electronics engineers, mathematicians and programmers. Six-axis CMM, using its main advantage - the six-axis control of the probe - are able to control the difficult to reach surface of products, to measure performance in a 2-5 times greater than traditional CMM.
For the current stage of development of precision engineering and instrumentation are relevant problem of increasing the precision, performance and reliability of the CMM. The solution of this problem is devoted to leading companies and scientists [1-17]. They generally, relate to the technological problems of building new types of measuring machines. At the moment, developers do not have the mathematical and information software, that allows on the stages of creation, operation and modernization, as well as in real time to analyze the mutual influence of the parameters of the CMM. These parameters are combined complex causal network and assess the impact on them of external factors and thereby optimize the design of perspective CMM is difficult.

This fact determines the relevance, practical and scientific significance as well as the economic feasibility to development of models and algorithms for improves the performance and accuracy of the six-axis CMMs.

\section{Characteristics of the Research Object}

Based on research findings required to develop software, which includes a complex of mathematical models.

\footnotetext{
* Corresponding author: zov17@mail.ru
} 
These models allow to perform simulation the dynamics of changes in the values of the basic parameters of the CMM, affecting the accuracy and performance.

The structure of the software need to include:

- graph of causal relationships;

- system of nonlinear differential equations;

- regression models for the formal description of the causal relationships between the parameters of the models;

- algorithms to verify the adequacy of the developed models;

- information and logic scheme, that characterizes the processes of practical use of software developed in the structural sub-divisions of engineering enterprises.

2.

In this article, we solve problems of paragraphs 1 and

Scheme of six-axis CMM is shown in Fig. 1. All process operating control factors on $\mathrm{CMM}$ can be divided into three groups: internal parameters CMM input actions and external influences. The Interior parameters are set for design of CMM and operational factors. During the measurement of these parameters may be constant or vary. Input exposure determined by the parameters of the measured product, measurement tasks and mathematical software. External influences vary in function of time and related to the environment, are mostly perturbing character. These groups of factors affect both the performance and the accuracy of control.

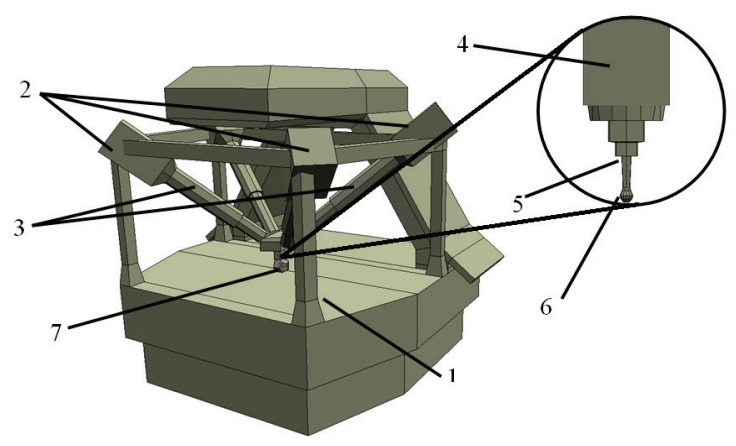

Fig. 1. Scheme of six-axis CMM: 1 - Table; 2 - Hinges; 3 Telescopic Rod; 4 - Base of the Touch Sensor; 5 - Touch Sensor (probe); 6 - Spherical Tip of the Touch Sensor; 7 Measured Product.

Performance $\mathrm{X} 1$ is the main parameter to be optimized CMM, which directly or indirectly affect all other parameters. By definition, the performance is an indicator of the efficiency of production, which characterizes output per unit of resources used, the factors of production. Therefore, performance of measurement will be determined by the following formula:

$$
X_{1}=\frac{V}{T \cdot k},
$$

where $V$ - volume of the measuring space, $\mathrm{mm} 3 ; T-$ time of measurement, sec; $k$ - coefficient of measurement complexity.
Accuracy $X_{2}$ is defined as the maximum error in the length measurement direction in parallel to the coordinate axes (ISO 10360-2):

$$
X_{2}=k_{0}+k_{1} L
$$

where $k_{0}$ - coefficient determined by the components of the measurement errors that are independent of measuring length (touch sensor error, electrical inertia, discontinuity and short-period errors coordinate displacements of nodes, errors associated with dynamic phenomena); $k_{1}-$ coefficient defined components, depending on the measured length $L$ (long-period measurement error and coordinate movement of nodes, the impact of ambient temperature, deformation coordinate displacement devices).

Requirements for the simultaneous increase of productivity and accuracy are contradictory in many cases. Thus, as a criterion for the optimization of control process must be productivity, which the accuracy demands attributed to limitations problem.

Other important factors when measuring on CMM:

$X_{3}$ - mechanical design error, $\mu \mathrm{m}$;

$X_{4}$ - structural rigidity, $\mathrm{N} / \mu \mathrm{m}$;

$X_{5}$ - error of the rotation hinges, rad;

$X_{6}$ - error linear displacement rods, $\mu \mathrm{m}$;

$X_{7}$ - error fixing the moment of contact, $\mu \mathrm{m}$;

$X_{8}-$ geometric accuracy of the spherical tip of the touch sensor, $\mu \mathrm{m}$;

$X_{9}-$ error of laser interferometers, $\mu \mathrm{m} / \mathrm{m}$;

$X 10$ - speed of linear motion along the axes, $\mathrm{mm} / \mathrm{s}$;

$X_{11}$ - speed of angular motion along the axes, $\mathrm{rad} / \mathrm{s}$;

$X_{12}$ - speed of rotation of the touch sensor; $\mathrm{rad} / \mathrm{s}$;

$X_{13}$ - calibration time, sec;

$X_{14}$ - application of the contactless scanning head, bool;

$X_{15}$ - dimensions of the measured product, $\mathrm{mm}$;

$X_{16}$ - length for probe of the touch sensor, $\mathrm{mm}$;

$X_{17}$ - time for installation of the product in the process control, sec;

$X_{18}$ - time of creation a mathematical model of the product, sec;

$X_{19}$ - number of measurements to align the coordinate systems of the measured product and the CMM;

$X_{20}$ - number of measurements at the control size;

$X_{21}$ - number of measurements in the control form;

$X_{22}$ - number of measurements at the control of deflection location for surface;

$X_{23}$ - number of measurements for further analysis;

$X_{24}$ - temperature fluctuation during measurement, degree/min;

$X_{25}$ - deformation of the base and the base parts of CMM, $\mu \mathrm{m}$.

\section{Mathematical Models}

Order to reflect of this interaction is built Count causal relationships shown in Fig. 2. 


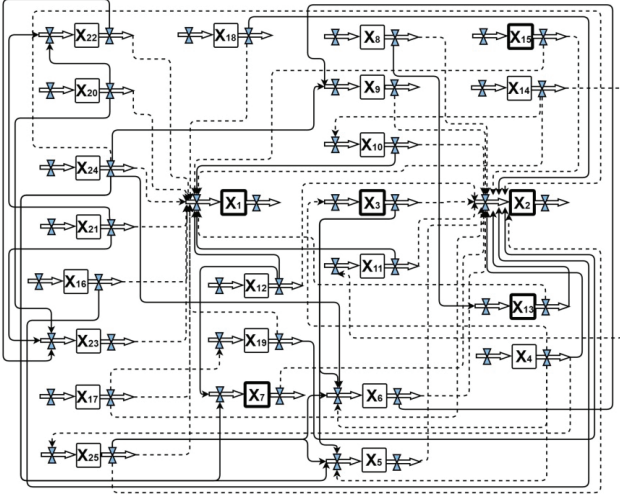

Fig. 2. Count causal relationships.

Based on the graph causal relationships to build a system of differential equations, each of which has the form:

$$
\frac{d X_{i}}{d t}=X_{i}^{+}-X_{i}^{-} ; \quad F_{i}^{+}(\bullet)=S_{i}^{+} \Pi_{i}^{+} ; \quad F_{i}^{-}(\bullet) \equiv S_{i}^{-} \Pi_{i}^{-},
$$

where $X_{i}-$ simulated characteristic; $S_{i}^{+}$and $\Pi_{i}^{+} \quad-$ respectively the sum of external factors and the product of positively influencing variables; $S_{i}^{-}$and $\Pi_{i}^{-} \quad-$ adversely affecting the growth of variable $X_{i}$.

Thus, for the preparation of the differential equations of system dynamics is used relationship:

$$
\frac{d X_{i}}{d t}=S_{i}^{+} \Pi_{i}^{+}-S_{i}^{-} \Pi_{i}^{-} .
$$

The system of differential equations used for system simulation of the main characteristics of the CMM, has the following form:

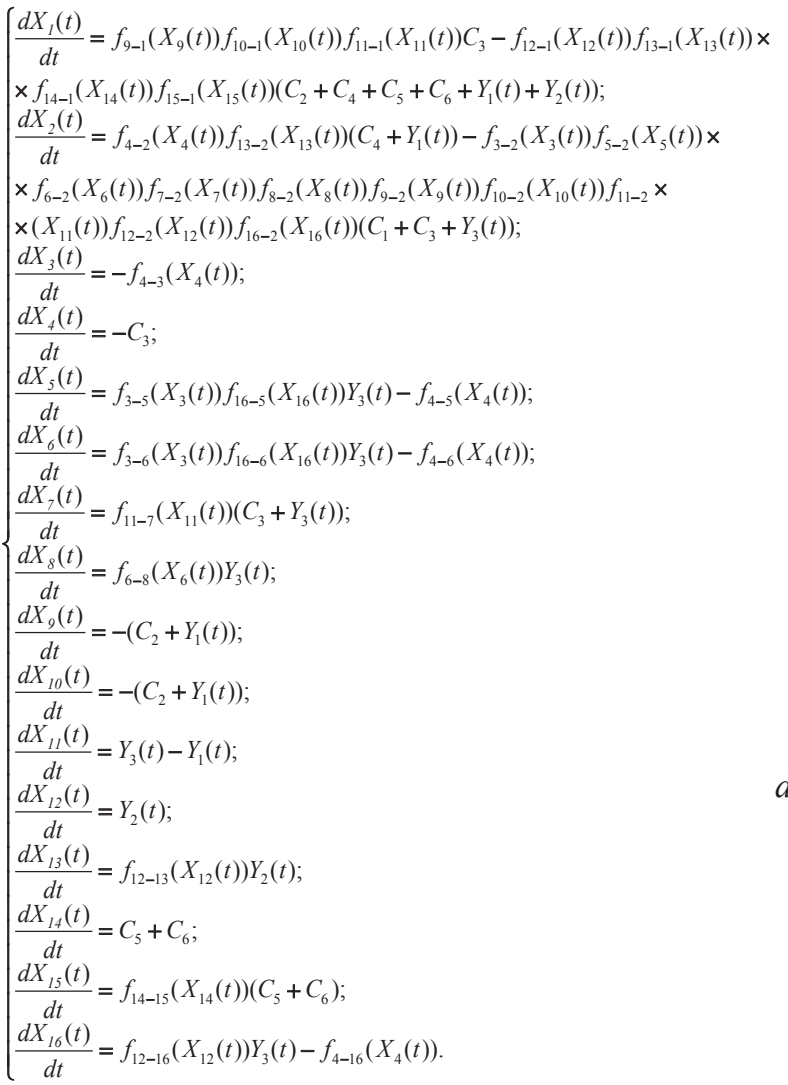

For the numerical solution of the system is necessary to normalize the parameters and set the initial values. After normalizing the simulated variables and substituting the functions $f_{i-j}$, the system takes the form:

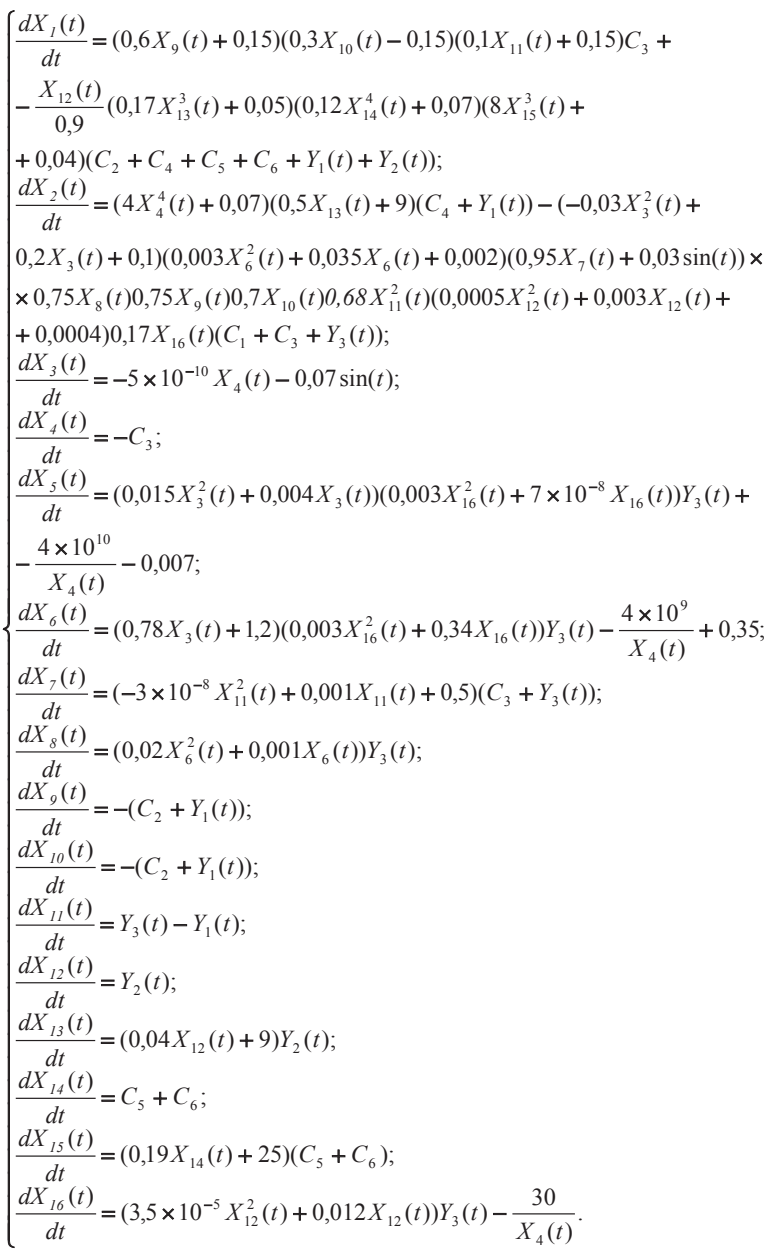

In developing this system of equations is used a statistical data describing the cause-effect relationship between the internal model parameters and environmental factors. It is shown the partial solutions for a model example at short time intervals in the form of cross sections performance and accuracy Hypersurfaces. These solutions make it possible to practically interpret developed models and methods of system dynamics used in solving the problem. It was found that a significant potential for growth performance while ensuring the specified accuracy of measurements is hidden in the optimization of the number, location and sequence of control points on difficult surfaces. Therefore, this direction is chosen as the most effective for further research. The results of these studies constitute the methodological basis for the development of new information and advising systems, which can be used to simulate and optimize the performance of existing and prospective Coordinate Measuring Machines.

\section{Conclusion}


Numerically solving systems of nonlinear differential equations allows you to find the optimal value of the performance under the constraints on the accuracy. The practical implementation of this approach can be used when creating the concept and appearance of perspective Coordinate Measuring Machines, possessing the highest accuracy, reliability and performance at this stage of technological development.

The study was performed by a grant from the Russian Science Foundation (project №16-19-10204).

\section{References}

1. B.S. Brazhkin and V.S. Mirotvorskii, Calculation of Curved Surfaces on Coordinate Measuring Machines, Measurement Techniques 48, pp. 657 (2003)

2. V.G. Domrachev, V.M. Grechishnikov and A.Zh. Chernyavskii, Determination of the Oscillation Parameters of the Blades of Turbine-Driven Sets based on Nonlinear Approximation of the Signals of Primary Converters, Measurement Techniques 56, pp. 1242 (2014)

3. O.A. Yalovoy, O.V. Zakharov and A.V. Kochetkov. The Centerless Measurement of Roundness with Optimal Adjustment, IOP Conf. Series: Materials Science and Engineering 93, pp. 012024 (2015)

4. O.V. Zakharov, I.N. Bobrovskij and A.V. Kochetkov, Analysis of Methods for Estimation of Machine Workpiece Roundness, Procedia Engineering 150, pp. 963 (2016)

5. M. Sieniło, Compensation of influence of elements eccentric positioning on the result of roundness deviation measurement of discontinuous sections by radial method, Advances in Intelligent Systems and Computing 393, pp. 361 (2016)

6. J. Caja, E. Gómez, P. Maresca and M. Berzal, Development of a Calibration Model for Optical Measuring Machines, Procedia Engineering 63, pp. 225 (2013)

7. S.H. Mian and A. Al-Ahmari, Enhance performance of inspection process on Coordinate Measuring Machine, Measurement 47, pp. 78 (2014)

8. F.A.M. Ferreira, J. de Vicente y Oliva and A.M.S. Perez, Evaluation of the performance of coordinate measuring machines in the industry, using calibrated artefacts, Procedia Engineering 63, pp. 659 (2013)

9. R. D'Amato, J. Caja, P. Maresca and E. Gómez, Use of coordinate measuring machine to measure angles by geometric characterization of perpendicular planes. Estimating uncertainty, Measurement 47, pp. 598 (2014)

10. A. Gąska, P. Gąska and M. Gruza, Simulation Model for Correction and Modeling of Probe Head Errors in Five-Axis Coordinate Systems, Appl. Sci. 6, pp. 144 (2016)
11. R. Calvo, R. D’Amato, E. Gómez and R. Domingo, Integration of Error Compensation of Coordinate Measuring Machines into Feature Measurement: Part II - Experimental Implementation, Sensors, 16 pp. 1705 (2016)

12. Q. Huang, K. Wu, C. Wang, R. Li, K.-C. Fan and Y. Fei, Development of an Abbe Error Free Micro Coordinate Measuring Machine, Appl. Sci. 6, pp. 97 (2016)

13. V.A. Pechenin, M.A. Bolotov, Basing error in coordinate measurements of cylindrical gears, Russian Engineering Research 36, pp. 630-634 (2016)

14. V.A. Pechenin, M.A. Bolotov, N.V. Ruzanov, Development of a method of ICP algorithm accuracy improvement during shaped profiles and surfaces control, International Journal of Engineering and Technology 6, pp. 2229-2235 (2014)

15. M.A. Badar, S. Raman and P. S. Pulat, Experimental verification of manufacturing error pattern and its utilization in form tolerance sampling, International Journal of Machine Tools and Manufacture 45, pp. 63-73 (2005)

16. Małgorzata Poniatowska and Andrzej Werner, Simulation tests of the method for determining a cad model of free-form surface deterministic deviations, Metrol. Meas. Syst., XIX, pp. 151-158 (2012)

17. R. Ascione, G. Moroni, S. Petrò and D. Romano, Adaptive inspection in coordinate metrology based on kriging models, Precision Engineering 37, pp. 4460 (2013) 